\title{
Ictericia colestásica del primer trimestre de vida
}

\author{
Eduardo Chávez C. ${ }^{1}$; Bárbara Pizarro M. ${ }^{1}$; Gladys Guevara P.1, 2; \\ Sergio Ceresa O. ; Jorge Las Heras B.,4; Elena Kakarieka W.4
}

\section{Cholestasis in the first three months of life}

The records of 5 ? palients with neonalol cholestasis, which were seen from jonvory 1980 throughoul july 1991 , were rewieved to stimulate timely diagnosis and therapy. Clinical fealues, laboratory findings and final diagnesis are described. Fitty three percent of the potienls were refered to the gaslioenlerology unit al ar after age two months even though $65 \%$ of the whole sample had acholia. The differentiation belween intrahepalic and extrahepatic, bile ducl, disease was based on histopatologic fealures, hepatic scirligrafy and evolution. Among forty two patients with intrahepatic cholestosis, twenty two hod idiopolic neonatal hepalilis ond twenly had specific etiologies. Of nine polienls with extrahepolic disease, eight had biliary alresia and the other one had an extrinsic compresion of the billiary free, caused by local and limph node inflanatory reaction. The oulcome of the palients with intrahepolic disease was solisfactory in 30 cases, which are already alive and without hepatic damnage. Only one of the nine palients wilh extrahepatic bile duct disease sunived at the end of this sludy.

¿Key words: jaurdice, obsluclive, cholestatic, extrahepalic, bile duct obstuction, intıhepalic; neonatali:

La ictericiá colestásica representa un dilcma diagnóstico para el médico, que debe decidir oportunamente, y en el plazo más corto posible, si la cnfermedad es atribuible a una causa de manejo médico o a una de resolución quirúrgica, pues la atresia de vía biliar ticne mejor pronóstico si se realiza portoenteroanastomosis antes de sesenta días de vida, la lista de causas potenciales es larga y diversa y la presentación clínica puede ser indistinguible en las diferentes formas etiologicas de la enfermedad 1 .

Con el propósito de estimular el estudio apropiado y la referencia adccuada de estos cnfermos, se describe la experiencia de una unidad de gastroenterología pediátrica con los pacientes alendidos entre enero de 1980 y junio de 1991 con dicho diagnóstico.

1. Unidad de Gastroenterologia Infantil, Servicio de Pediatría. Hospital Clínico San Borja Arriarần.

2. Departamento de Pediatría Centro, Facultad de Medici na, Universidad de Chile.

3. Unidad de Anatomia Patológica, Campus Centro, Facultad de Medicina. Universidad de Chile.

4. Servicio de Anatonnáa Patológica, Hospital Clínico San Borja Amiarán.

\section{Material y Métodos}

Sc revisaron las fichas de 51 pacientes atendidos en la unidad de gastroenterologia del servicio de pediatría del hospital San Borja Arriarán, mediante un formulario previamente diseñado en el que se registraton la cdad de la primera consulta en la unidad, edad gestacional, peso de nacimiento, sexo, presencia de acolia y coluria, esplenomegalia, bilirrubina total y directa, transaminasas, aljafetoproteína, gamnaglutamiltranferasa (GGT); serología para identificación de infecciones for 1oxoplasma, vins de nubéola, citomegalovirus, herpes virus, virus hepatitis $B$, tripanosomiasis (Chagas); estudio de enfermcdades metabólicas: aminoacidemia aminoaciduria, sustancias reductoras en orina, además de los resulıados histopatológicos, cintigráficos y de ecografía de hígado y vías biliares. Se exclu. yeron de este análisis los casos de colestasia secundarios a alimentación parenteral. Los resultados se expresaron cn porcentajes, promedio y medianas. La clasificación definitiva de la colestasia en intra y extuahepática se bas 6 en la evolución clínica, la cintigrafía y la biopsia hepática. Esıa última no se realizó cuando se registraba evidencia de reducción de la colestasia o mejoría de las manifestaciones clínicas.

\section{Resultados}

En la tabla 1 se describen las características clinicas de los 51 pacientes estudiados, $42(82,4 \%)$ de to cuales eran varones. Entre los cuarenta y 
Tabla 1

Ictericia colestásica del lactante menor. Características clínicas

\begin{tabular}{|c|c|c|c|}
\hline & \multicolumn{2}{|c|}{ CIH } & \multirow{2}{*}{$\begin{array}{l}\text { CEH } \\
\mathrm{n} \quad(\mathscr{\%})\end{array}$} \\
\hline & $\mathbf{n}$ & $(\%)$ & \\
\hline Total & 42 & & 9 \\
\hline $\begin{array}{l}\text { Sexo: masculino } \\
\text { femerino }\end{array}$ & & $\begin{array}{l}(71,4) \\
(28,6)\end{array}$ & $\begin{array}{l}2(22,2) \\
7(77,8)\end{array}$ \\
\hline $\begin{array}{l}\text { Edad de consulta: } \\
>\text { de } 42 \text { días }\end{array}$ & 30 & $(71,4)$ & $8(88,9$ \\
\hline Peso de nacimiento & & & \\
\hline $1000-2499$ & 21 & $(27,5)$ & $1(11$ \\
\hline 2500 o más & 29 & $(72,5)$ & $8(88.9)$ \\
\hline Acolia persistente & $22 / 39$ & $(56,4)$ & $9(100)$ \\
\hline Esplenomegalia & $23 / 39$ & $(58.9)$ & $4(44$ \\
\hline
\end{tabular}

CIH: colestasia intrahepática.

CEH: colestasia extrahepatica.

dos pacientes definitivamente calificados como colestasias intrahepáticas la mayoría eran varones, en cambio en los nueve afectados por atresia de vías biliares (extrahepáticas) predominaron tas mujeres. En doce casos $(23,5 \%)$ el peso al nacer fue menor de $2500 \mathrm{~g}$, en once de ellos la obstrucción era intrahepática. Treinta y ocho niños $(74,5 \%)$ fueron enviados a consultar después de seis scmanas de edad, 31 de 49 nifios en que se registró el antecedente $(63,3 \%)$ tenían acolia persistente, incluyendo todos los afectados por obstrucción cxtrahepática y algo más de la mitad de los que sufrian obstrucciones intrahepáticas. En dos pacientes no se consignó el color de las deposiciones en la historia.

En la tabla 2 se muestran los resultados de algunos de los exámenes bioquímicos de laboratorio, cintigrafía, ecografía y biopsia hepática, destacando la ausencia de paso de marcador al intestino en los 8 casos de obstrucción extrahepática estudiados por este método y en 8 de 17 niños con colestasias intrahcpálicas, asi como la importancia que tiene la biopsia hepática en el diagnóstico. En la tabla 3 se expresa la distribución de los diagnósticos finales formulados, donde destaca el franco predominio del síndrome de hepatitis neonatal entre los casos de colestasia intrabepática y que, con una sola excepción en que habia compresión cxtrínseca por una masa ganglionar, la mayoría de las extrahepáticas co-

Tabla 2

Ictericia del lactante menor: exámencs

\begin{tabular}{|c|c|c|c|}
\hline & $\mathrm{CIH}$ & CEH & \\
\hline Total & 42 & 9 & \\
\hline \multicolumn{4}{|l|}{ Exămenes de laboratorio: } \\
\hline Bjirrubinemia total (mg\%) & 8,4 & 9,6 & \\
\hline Bilirrubinemia directa (\%) & 61.4 & 59,7 & \\
\hline SGOT (UI/) & 144,6 & 168,0 & \\
\hline Alfafetoproteina $(\mathrm{mg} / \mathrm{ml})$ & 6971 & 6177 & $(4)^{*}$ \\
\hline Gamaglutamil transferasa (U/1) & 212,2 (19) & 284 & $(6)^{*}$ \\
\hline \multicolumn{4}{|l|}{ Cintigrafia hepática (25) } \\
\hline Con paso a intestino & 9 & 0 & \\
\hline Sin paso a intestino & 8 & 8 & \\
\hline \multicolumn{4}{|l|}{ Ecografía (36) } \\
\hline Normal & 24 & 5 & \\
\hline Dilatación quística de colédoco & - & 1 & \\
\hline No concluyente & 6 & - & \\
\hline \multicolumn{4}{|l|}{ Biopsia hepática (31) } \\
\hline Sugerente de hepatitis & 17 & 1 & \\
\hline Sugerente de alresia & 1 & 8 & \\
\hline Hipoplasia de conductillos & 3 & - & \\
\hline Hepatitis con atresia & 1 & - & \\
\hline
\end{tabular}

* Entre paréntesis el total de pacientes en los cuales se realizô el examen. 
Tabla 3

Ictericia del lactante menor: diagnósticos finales

CIH (n: 42)

S. hepautis neonatal

Citomegalovirus

Rubéola

Hepatitis B

Toxoplasmosis

Chagas

Lúes

Hepatitis séplica

Hipoliroidismo

Tirosinemia

Hipoplasia de conductillos

CEH (n: 9)

Atresia biliar

Plastrón ganglionar

* En 3 casos se asoció a dilaración quística del colédoco.

\section{Tábla 4}

Ictericia colestásica del lackante menor: crolución

\begin{tabular}{lcc} 
& CIH & CEH \\
Vivo sin daño hepático & 30 & $-{ }^{-}$ \\
Vivo con daño hepático & - & 1 \\
Fallecidos & 4 & 5 \\
Indeterminados & $8 *$ & $3^{* *}$ \\
\hline
\end{tabular}

* Devueltos a hospital de origen

** Dos pacientes se envían para cirugía a olro hospital, uno fue devuelto a su bospital de origen.

mespondía a atresia de la vía biliar, que en toes casos se acompañaba de dilatación quistica del colédoco. En tabla 4 se describe la evolución clínica de estos pacientes, que en 11 casos no fue posible determinar, ures de cllos con lesiones extrahepáticas. De los pacientes con las formas intrahepáticas, 30 experimentan mejoría clínica y de laboratorio, 4 pacicntes fallecieron, en 8 no se pudo precisar evolución (4 de ćstos ya tenían cirrosis al momento del diagnóstico). De los pacicntes con colestasia extrahepática uno fue considerado fuera de alcance quitúrgico, fallociendo a los ocho meses de edad; tres fueron derivados a otro centro asistencial para reparación quirúrgi- ca y cinco fueron intervenidos en nucstro hospjtal. En todos estos úlumos pacientes se confirmó la ex istencia de atreșia de la vía biliar y se realizó un procedimiento de Kasai. Uno de ellos sobre. vivió y fue devuelto a su hospital de origen, los otros cuatro fallecieron, tres en el postoperatorio inmediato y uno-operado a los tres meses de vida- a la edad de 2,5 años.

\section{Comentario}

En esta serie la mayoría de los pacientes fueron referidos a los especialistas después de la edad de sescria días, lo que perjudica su pronóstico y justifica una labor de sensibilización, para lograr la derivación precoz desde el nivel de atención primaria, cvizando retrasos al tratamiento operatorio ${ }^{6.7}$.

Sc ha señalado que, por razones no definidas, la hepatitis neonatal parece ser más común en varones, especialmente en ayuellos nacidos prematuramente o con bajo paso de nacimiento, en contraste con la atresia de vía biliar extrahepática que ocurre más frecucntemente en niñas, $1,8,9$, como se confirma en nuestra experiencia. La proporción de niños con peso al nacer inferior a $2500 \mathrm{~g}$ entre las colestasias intrahepáticas es inferior, sin cmbargo, a la esperada.

La observación de las deposiciones como parte del examen físico ha sido considerada de gran importancia, ya que deposiciones acólicas persistentes indicarían un al to grado de obstrucción ${ }^{1}$. 10-12. Las deposiciones coloreadas permiticion orientar cl estudio a causas intrahepáticas, puesto que todos fos pacientes con colestosia extrahepática tenían acolia al momento del diagnóstico, aun cuando este fenómeno tarnbién fue observado en la milad de los que tenían lesiones intrahepáticas. Llama la atención que en dos casos de la seric no se consignó cste importante dato en la historia, lo que obliga a insistir en la necesidad de no omitirlo.

La esplenomegalia, considerada generalmente asociada con infecciones congénitas, enfermedades de depósito, enfermedad hemolítica, entre otras ${ }^{11}$, fue detectada en más de la mitad de los casos de colestasia intrahepática y en proporción algo menor de pacientes con obstrucción exirahepática, por lo que no fue de ayuda en cl diagnóstico diferencial. La presencia de coluria no fue tabulada en este trabajo, dado que 
sólo deternina la presencia de colestasia y por definición la presentaban todos estos enfermos.

En un estudio de seguimiento en 56 pacientes con hepatitis neonatal, en la Universidad de Taiwán, China, consideraron que valores mayores de $15 \mathrm{mg} / \mathrm{dl}$ de bilirrubina total y $10 \mathrm{mg} / \mathrm{dl}$ de bilimubina directa eran signos de peor pronóstico $^{13}$. Por otra parte, Ferry y Selbi, de la Universidad de Baylor, Texas, no encontraron diferencia en los valores de bilirrubina al comparar colestasias intra y extrahepáticas ${ }^{3}$, 10 que coincide con lo observado por nosotros. Los valores de transaminasas, de alfafetoproteina y gamma glutamil transferasa no ayudaron en el diagnóstico diferencial, lo que ha sido mencionado por otros autores $3,4,8$,

La cintigrafía hepatobiliar con Tc 99 unido a ácido diisopropyl iıninodiacético (DISIDA) es un procedimiento útil en la detección de la permeabilidad del árbol biliar ${ }^{12-20}$. En esta serie identificó correctamente a todos los casos de colestasia extrahepática en que se emplé la prueba; sin embargo, en la mitad de los pacientes con la forma intrahepática fue también sugerente de obstrucción completa. Es importante hacer notar que el paso del radiofármaco hacia cl intestino excluye una obstrucción total del tracto biliar al momento del examen ${ }^{3}$. Por esta razón es tan conveniente el cmpleo de la biopsia hepática como estudio complementario en estos pacientes $y$, además, no postergar la laparotomia más allá de la edad de 60 dias.

La ecografía abdominal realizada después de 3 a 4 horas de ayuno, según algunos autores $1,8,9,11$. 18, 26, 21 pemitiría la cvaluación del higado y del árbol biliar. La dilatación anormal de conductos biliares y la dificultad para yisualizar la vesícula biliar ayuda a sospechar atresia de vía biliar. Sin cmbargo, donde mayor valor tiene es en la detección de la dilatación quística del colédoco asociada a atrasia de la vía biliar. El valor de este procedimiento fue limitado en la serie que se analiza, probablemente porque nuestro grupo aún no había acumulado suficiente experiencia en esta materia en el período que abarca esta observación.

La biopsia hepática es aplicable en cualquier centro hospitalario de tipo A, ticne bajo costo y prácticamente carece de inconvenientes para el paciente. La interpretación por un patólogo experimentado puede hacer el diagnóstico correc- tamente en 90 a 95\% de los casos 1 3.8, 18.21-23. En nuesta scrie fue el examen de mayor utilidad para diferenciar colestasias intra y extrahepálicas cuando la evolución clínica, la coografía y la cintigrafía no permitían hacerlo en conjunto.

La etiología de colestasia intrahepática, al igual que lo descrito en la literatura, fue más frecuentemente calificada como hepatitis neonatal idiopática, en menor grado se identificaron causas como citomegalovirus, enfermedad de Chagas, rubéola, hepatitis B, toxoplasmosis, lúes, hepatitis séptica, hipotiroidismo, tirosinemia e hipoplasia de conductillos 1, 2, 4,21,24. En los casos de atresia de vías biliares destaca la asociación con dilatación quística del colédoco en tres pacientes, lo que coincide con lo descrito por Landing en su teoría de la colangiopatía obstructiva de la infancia22.

La mala evolución de nuestros pacientes con colestasia extrahepálica y la baja frecuencia de esta afección (9 casos en 11 años) nos sugiere que cl manejo quirúrgico debiera efectuarse en centros que concentren este tipo de patología, adquiriendo así una experiencia más sólida cn la técnica quirúrgica y manejo pre y postoperatorio. Así también, dado el escaso número de pacientes, nos parece necesaria la realización de protocolos de cstudio y seguimiento comunes en los diferentes centros gastroenterológicos pediátricos, para alcanzar conclusiones más significalivas.

\section{Resumen}

Se describen los hechos clínicos, de laboratorio y el diagnóstico final de 51 pacientes con ictericia colestâsica del primer trimestre de la vida, observados en un hospital metropolitano de Santiago entre enero de 1980 y junio de 1991. Cincuenta y tres por ciento de los pacientes fucron enviados al especialista con dos o más meses de edad. En $65 \%$ de los casos había acolia, incluyendo los nueve con diagnóstico definitivo de obstrucción extrahepática y más de la milad de los catalogados como obstrucciones intrahepáticas. La diferenciación entre colestasia intrahepática y extrahepática se basó en critcrios histopatológicos, cintigráfícos y evolutivos. De 42 pacientes con la forma intrahepática 22 correspondieron a hepatitis neonatal idiopática y el resto a causas específicas. En ocho de las coles- 
tasias extrahepáticas se confirmố atresia de vías biliares y un caso compresión de vía biliar por plastrón ganglionar. La evolución de los pacientes con colestasia intrahepática fue buena en 30 (sin daffo hepático). En la forma extrahepática 8 pacientes han fallecido al cerrar este estudio, siendo el único sobreviviente un niño portador de atresia de las vías biliares sometido a una operación de derivación de Kasai.

(Palabras clave: ictericia, obstructiva, colestásica, extrahepática, intrahepática, neonatal, primer trimestre.)

\section{Agradecimientos}

Al Dr. Julio Espinoza por la revisión crítica de este manuscrito.

\section{Referencias}

1. Balistreri W: Neonatal Cholestasis. J Pediatc 1985; I06: $171-183$.

2. Momal A: Hepatitis and Choleslasis in Infancy. En: Mowat A. ed. Liver disorders in Childhood, London: Butterworths, 1983; 37.71.

3. Ferry G, Selby M, Udall, Finegold M. Nichols B: Guide to Early Diagnosis of Biliary Obstruction in Infancy. Clin Pediatr 1985; 24: 305-311.

4. Silverman A, Roy $C$. Prolonged Obstructive jaundice. Ped Clin Gastroenterol $3^{\circ}$ edition. The CV. Mosby Co., Sı Louis. 1983; 494.556.

5. Kobayashi A, Irabashi $F$, Ohbe $Y$ : Long-tem prognosis in biliary atresia after hepatic portoenterostony: Analysis of 35 patiens who survived beyond 5 years of age. J Pediatt 1984; 105: 243-245.

6. Mieli-Vergani G, Howard E. Poriman B, Mawat A: Late Referral for Biliary Atresia-Missed Oportunities for Efective Surgery. Lancet 1989; i; $421-423$.

7. Kasai M, Smzuki H, Ohoshe E: Technique and results of operative management of biliary atresia. World I Surg 1978; 2: 571-580.
8. Burron E, Babock D, Heubi J, Caelfound M: Neonalal jaundice. south Med J 1990; $83: 294$-302

9. Karrer, Helir. Stewari B. Lilly J. Congenital Biliary Tract Disease. Surgical Clin of North Am 1990; 70: 1403-1418.

10. Greene H, Helinek $G$, Morán $R, O$ Neil J: A diaguostic approach to prolonged obstructive jatundice by 29 hours collection of duodenal fluid. J Pediatr 1979; 95 : 412414.

11. Fitzgerald J: Cholestatic Disorders of Infancy. Pediatr Clin North Am 1988; $35:$ 357-373.

12. Brown $S$, Househand $K$ : visual stool examination-a screening test for infants with prolonged neonalal cholestasis. S Afr Med J 1990; 77: 358-359.

13. Chang M, Hsu H, Lee Ch, Wang T, Kao Ch: Neonatal Hepatitis: A follow-up study. J Pediaur Gastroenterol Nu1r 1987; 6: 203-207.

14. Betancourt $F$, Larrafn $F$, Gonzailez $P$ et al. Cintugrafía de la váa biliar en el Síndrome xctérico del lactante menor. Rey Chil Pediatr 1983; 54: 237.240.

15. Gerhold J, Klingensmith W. Kuni Chet al.: Diagnosis of biliary atresia with radionuclide hepatobiliary umaging. Radiology 1983; 146; 499-504.

16. Alman $R$, Levy $J$ : Biliary Alresin. Pediatr Ann 1985; $14: 481.485$.

17. Rasentall L, Shaffer $E$, Lisbana $R$, Pare P: Diagnosis of hepalobiliary disea se by 99 1c-HIDA cholescintir.grafy. Radiology 1978; 126: 467-474.

18. Kurks D, Coleman E. Filston $H$ el al.: An imaging approach to persistent neonatal jaundice. AJR 1984; 142: 461-465.

19. Hung W, Sk Ch: Diagnosis of atretic prolonged obsIructive jaundice; Techntium $99 \mathrm{~m}$ hepatolite excretion study. Pediatr Sur 1990; 25: 797-800.

20. Torr issi J, Hallen, Veicek F: Choledocal cyst and biliary atresia in the neonate. Imaging Funding in fje cases. AJR 1990; 155: 1273-1276.

21. Watson S, Giacoin: Cholesiasis in Infancy. Clin Pediatr $1983 ; 22: 30-35$.

22. Landing B: Considerations of the Pathogenesis of Neonatal Hepatitis, Biliary Atresia and Choledocal Cyst - The concept of Infantile Obsinuctive Cholangiopahy. Prog Pediat Surg 1974; 6: 113-139.

23. Danus $O$, Larrain $F$, Lutorre $J$ : Colestasia cronica de] lactante menor. Rev Chil Pediatr 1984; 55 : $398-401$.

24. Ceresa S, Rossel M, Las Heras J. Toro L, Curotto D. Hepatitis neonatal por virus Rubeola. Rev Chil Pediatr 1983; 54: 424-427. 